\title{
DIAGNOSIS AND TREATMENT OF ADHD: NIH CONSENSUS REPORT
}

The following is a synopsis of the consensus statement issued by the planning committee and panel members of a conference on ADHD held at the NIH, November $16-18,1998$. Of 44 panel members, 13 were psychiatrists, 10 psychologists, 7 pediatricians, 4 educators, 4 government, DEA, and consumer agency representatives, 2 pharmacologists, 2 statisticians, 1 juvenile justice, and 1 pediatric neurologist. The conclusions were as follows:

- ADHD represents a major public health problem.

- The use of stimulants creates a major controversy.

- No independent diagnostic test is available, and further work is needed to determine specific diagnostic criteria.

- Health care and social service agencies need an integrated approach to management.

- More long-term trials of treatment strategies are needed.

- A threshold for treatment with stimulants should be set.

- A consistent set of practice guidelines is required.

- Causes are speculative, and we have no strategies for prevention.

Published in IAm Acad Child Adolesc Psychiatry February 2000;39:182-193.

(Respond: John H Ferguson, MD, National Institutes of Health, 31 Center Drive, MSC-2082, Bethesda, MD 20892). Also with Commentary (Jensen PS. The NIH ADHD consensus statement: Win, lose, or draw? I Am Acad Child Adolesc Psychiatry February 2000;39:194-197).

COMMENT. Despite the conclusion and admission that ADHD has a central nervous system basis, the composition of this panel is surprisingly deficient in child neurologists and neuroscientists. The data from neurological, neuropsychological, electrophysiological, neuroimaging, and now genetic studies should command more attention. The conclusions reached by the panel are discouraging, and suggest the need for a change in approach and emphasis, in both basic and clinical research in ADHD. Symptom-based lists of diagnostic criteria, and their frequent DSM modifications, should be supplemented with more objective signs, including evidence of neurologic and perceptual dysfunction. Studies emphasizing etiology and prevention should be promoted in preference to symptom classification and drug trials. The exciting developments in the fields of SPECT, neuroimaging, and genetics, at present experimental, could be applied selectively in diagnosis, management and development of new treatments.

The Scandinavian syndrome of DAMP (deficits in attention, motor control and perception) (Landgren M et al. Dev Med Child Neurol 1996;38:891-906), like that of MBD in the US (minimal brain dysfunction), emphasizes the association of neurologic signs of motor dysfunction, perceptual dysfunction, and attention deficits, whereas the current American DSM criteria for ADHD include only symptoms of inattentiveness, hyperactivity, and impulsivity, excluding reference to tests for neurological and perceptual dysfunction. The overlap of these syndromes and the higher prevalence of DAMP compared to ADHD suggests that the neurologic examination and tests for perceptual dysfunction should form an integral part of the criteria for diagnosis of "attention deficit disorders." A return to the former minimal brain dysfunction criteria, in addition to the symptoms of ADHD, would allow a more objective diagnosis and earlier recognition and remediation of associated motor incoordination and perceptual deficits. For a neurological approach to ADHD see: Millichap JG. Attention Deficit Hyperactivity and Learning Disorders. Chicago, PNB Publishers, 1998. 